\title{
Risk Factors Associated with Invasive Cervical Carcinoma among Women Attending Jimma University Specialized Hospital, Southwest Ethiopia: A Case Control Study
}

\author{
Mesele Bezabih $^{1}$, Fasil Tessema ${ }^{2}$, Hailemariam Sengi ${ }^{3}$, Amare Deribew $^{4}$
}

\begin{abstract}
BACKGROUND: Cervical cancer is a more serious public health problem than other cancers in women in Sub-Saharan Africa in general and in Ethiopia in particular. Thus, this study assessed risk factors related to invasive cervical carcinomas in southwestern Ethiopia.

METHODS: Unmatched case control study was conducted in Jimma University Specialized Hospital from April 1 to September 30, 2010. The study consisted of 60 cases (women who had cervical cancers based on histopathologic examination) and 120 controls (women with no cervical cancers). Semistructured questionnaire was utilized for data collection. Vaginal examinations often visualized with speculum insertions were done for both cases and controls. Punch cervical biopsies were then performed for the suspected cases at Jimma University Hospital that serves about 15 million people in a catchment radius of $250 \mathrm{kms}$. Data were analyzed using SPSS version 13.0 software. Univariate and multivariate analyes were done to describe and identify independent predictors of cervical cancer.

RESULTS: The mean ages of cases and controls were $47.7(S D=10.8)$ and $35.5(S D=10.5)$ years respectively. Older women (40-59 years), $(\mathrm{OR}=4.7$; 95\% CI= 2.3-9.6), more than one husband (OR= 2.0; 95\%CI=1.0-3.9), as well as more than one wife in lifetime, $(O R=3.0 ; 95 \% C I=1.5-5.9)$, women who had more than 4 children, $(O R=10.3,95 \% C I=3.6-29.0)$, and age greater than 25 years at first full term delivery, $(O R=8.8 ; 95 \% C I=3.5-22.0)$ were statistically significant and the latter two were independently associated with invasive cervical cancer. Only 7(11.7\%) of cases and 58(48.3\%) of controls ever heard of cervical cancers; however, 2(3.3\%) of cases and 7(5.8\%) of controls had ever had history of papaneocolous (pap) smear tests done.

CONCLUSION: Poor knowledge on cervical cancer was observed that required more work to be done to increase knowledge of mothers on cervical cancer and on associated risk factors. Behavioral communication activities and establishment of cervical cancer screening programs for the young could help reduce the advancement of cervical cancer particularly among the less knowledgeable, older and grand multiparous women in our parts of the world.

KEYWORDS: invasive cervical carcinoma, risk factors, Jimma, south west Ethiopia
\end{abstract}

DOI:http://dx.doi.org/10.4314/ejhs.v25i4.8

\section{INTRODUCTION}

Cervical cancer as the most common cancer in women in developing countries is a preventable and curable disease, preventable by vaccination and screening and curable if identified at an early enough stage. It is gradually becoming a rare disease in many developed countries; this is not the case with many countries in sub-Saharan Africa. Cervical cancer is the most common cancer in women in sub-Saharan Africa and accounts for $22.2 \%$ of all cancers in women and it is also the most common cause of cancer death

\footnotetext{
${ }^{1}$ Department of Medical laboratory Sciences and Pathology, Jimma University, Ethiopia

${ }^{2,4}$ Department of Epidemiology and Biostatistics, Jimma University, Ethiopia

${ }^{3}$ Department of Obstetrics and Gynecology, Jimma University, Ethiopia

*Corresponding Author: Hailemariam Sengi, Email: hmullu@ yahoo.com
} 
among Sub-Saharan countries. Women in SubSaharan countries lose many years to cervical cancer than to any other type of cancer. Unfortunately, it affects them at a time of life when they are critical to the social and economic stability of their families (1,2). Developing countries contribute more than three-quarter of women death in cervical cancer every year and Ethiopia contributes substantially to this figure due to inadequate health care and virtually nonexistent screening mechanisms for early detection (3).

The cause of invasive cervical cancer has been the subject of numerous studies over the last 50 years. However, most of the epidemiologic research has been done in developed countries where cervical cancer incidence has declined significantly in the last three decades. Africa is a high-risk area for invasive cervical cancer, but few studies have analyzed the role of risk factors (4). Cervical cancer is also the most common cancer in women in Ethiopia. The average age specific incidence rate (for cervical cancer) for subSaharan countries is $31.0 / 100.000$ populations but, for Ethiopia, it is $35.5 / 100,000$ populations (1). As a result, this health problem brings to bear graver morbidity and mortality in Ethiopian women. Even though high-risk human papilloma virus (HPV) infection is a necessary cause, only a small proportion of HPV infected women develop invasive cancer. Hence, there must be co-factors to HPV infection that lead to the development of cervical cancer, and there is limited evidence about the extent of which of these cofactors are prevalent in developing countries like ours $(1,2)$.

Thus, the purpose of this study was to identify risk factors associated with invasive cervical cancers among mothers who attended Jimma University Specialized Hospital (JUSH) in Southwestern Ethiopia.

\section{METHODS}

This study was undertaken at JUSH in Jimma town located $352 \mathrm{~km}$ Southwest of Addis Ababa. Jimma is the main town in Southwestern Ethiopia with an estimated population of 120,000 (5). The JUSH is serving more than 15 million people in the Southwestern part of the country. Cervical cancer specimens taken in the gynecologic clinic were sent to the Pathology Unit within JUSH for histopathologic diagnoses. Data collection was performed from April 1 to September 30, 2010.

All women diagnosed with invasive cervical carcinomas were considered as cases and those without any evidence of invasive cervical carcinomas were recruited as controls. Inclusion criterion for cases was those classified as incident invasive cervical cancers based on hospital pathology reports. The diagnostic criterion for the cases was described microscopically as cervical cancers that breached or broke cervical epithelial tissue basement membrane and invaded the nearby subepithelial tissues (6). Whereas controls were those individuals who attended gynecologic clinic for any illness other than cervical cancers. The control patients were screened for not having any evidence of cervical cancers macroscopically on vaginal examination with speculum visualization. PAP smear test could not be preformed for lack of the badly needed reagents in the country at the time of the study and the test has never been practised in the southwestern part of the country.

Those cervical lesions including benign cervical neoplasms as well as non-invasive cervical carcinomas/cervical intraepithelial neoplasias (CIN), non-epithelial cervical cancers (sarcomas, etc) and metastatic cancers to the cervix uteri were excluded from cases. Exclusion criteria for controls were those less than 20 years of age and terminally ill patients.

The sample size for this unmatched casecontrol study was calculated by using WinPEPI (statistical software for epidemiologists) based on 95\% confidence level, $80 \%$ power, proportion of controls who had 5 or more parity (63\%) (7), odds ratio of 3.0 and case to control ratio of 1:2. Thus, this gave a total sample size of 180 individuals (60 cases and 120 controls).

For a case identified at the Gnecology and obstetrics Department in JUSH, two consecutive controls were recruited into the study until the required sample size was met.

Age, residence area, occupation, number of partners in life, parity, nonviable delivery, age at first full term delivery, prescence of sexually transmitted infections, age of first sexual intercourse, age of menarche and menopausal status (abscence of menustration for at least one year) were considered as possible risk factors in our study area. 
Semi-structured questionnaire was used for data collection. Two gynecology resident doctors and two clinical nurses having previous experiences in data collection filled the questionnaires through face-to-face interview and performed cervical specimens for the suspected cases to be delivered to pathology unit. A supervisor oversaw data collection for proper administration and the principal investigator verified serial case identifications and selection of controls weekly.

Cervical tissue specimens were delivered from obstetrics and Gynecology Department to the Pathology Unit in the Department of Medical laboratory sciences and pathology. The bottle labeling containing the cervical specimens and the respective request form were crosschecked for sameness. The tissues were also checked as a routine activity by pathology laboratory assistants for well fixation with formaldehyde fixative and the cervical specimens were then put in a tissue cassette where it underwent dehydration, clearing and impregnation steps within automatic tissue processor. Subsequently, tissue was conveyed to the paraffin wax for embedding. The specimen was then cut to a $4-5 \mu \mathrm{m}$ thickness with microtome machine and the sectioned ribbons were flattened onto water bath and finally put onto a dried clean slide where staining with hematoxylin and eosin procedures were undertaken prior to visualization under light microscopy.

The data were cleaned, coded and entered into a computer with the help of SPSS version 13.0 statistical software. Descriptive and bivariate analyses using Chi-square test and odds ratio were performed at the preliminary stage and multivariable binary logistic regression with backward stepwise method was implemented to identify independent factors. P-value less than 0.05 is used to declare statistical significnce.
The ethical clearance committee of the College of Public Health and Medical Sciences, Jimma University, reviewed the proposal and issued ethical clearance. In addition, support letters were obtained from the Clinical Director of JUSH and the Head of Gynecology and Obstetrics Department. Study participants were recruited into the study on voluntary basis with verbal consent. The procedure of this study per se is a routine activity done in aseptic and orderly manners, and thus, there were no risks to the participants. The information obtained from the respondents remained confidential.

\section{RESULTS}

Socio-demographic characteristics and findings: One hundred and eighty study participants (60 cases and 120 controls) were recruited in the study. The mean ages were 47.7 years $\mathrm{SD}=10.8$ and 35.5 years $\mathrm{SD}=10.5$ years for cases and controls respectively. Older women (40-59 years) were four times more likely to develop cervical cancer than those less than 40 years $(\mathrm{OR}=4.3 ; 95 \% \mathrm{CI}=1.8-10.2)$. Forty-five $(75.0 \%)$ cases and $67(67.3 \%)$ controls were rural residents, and area of residence had no association with cervical cancer $(\mathrm{OR}=0.85 ; 95 \% \mathrm{CI}=0.32$ 2.2). Most cases 52(86.7\%) and controls $114(95.0 \%)$ were married. Moreover, history of more than one husband in a lifetime was documented more commonly among cases, 24(40.0\%), than controls, $25(20.8 \%)$, and the crude odds ratio showed association $(\mathrm{OR}=2.0$; (95\% CI=1.0-3.9). On the other hand, 28(46.7\%) of the cases' and $26(21.7 \%)$ of controls' husbands had more than one wife in lifetime $(\mathrm{OR}=2.6 ; 95 \%$ $\mathrm{CI}=1.1-6.6)$. The median income for the cases was 840 Birr per month, ranging 100-3100; and for controls 1000.00 Birr/month, ranging 100-8000. By then one United States Dollar [USD]= about 17 Birr $)(\mathrm{OR}=0.87 ; 95 \% \mathrm{CI}=0.47-1.6)($ Table1 $)$.

Table 1: Sociodemographic variables, case control study, JUSH 


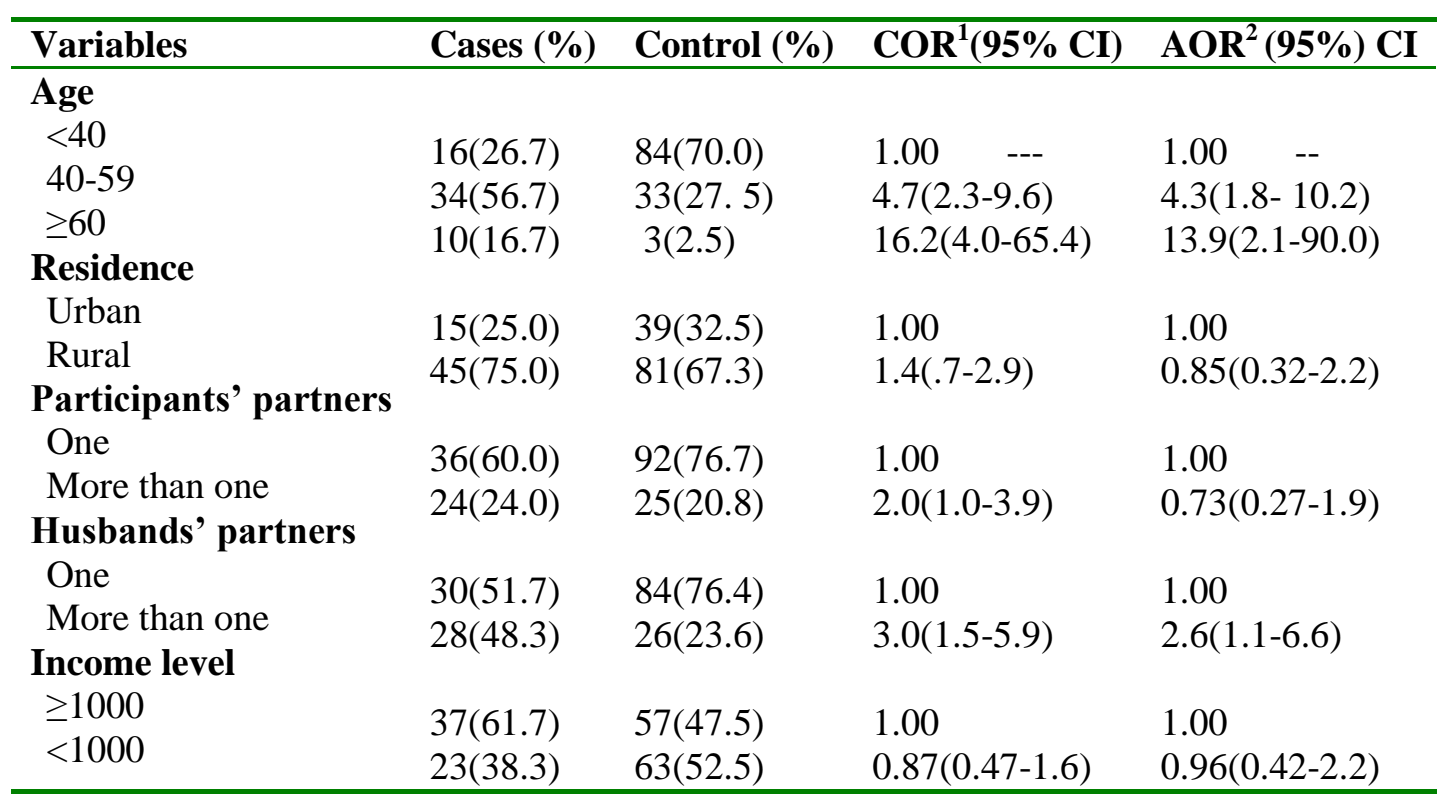

${ }^{1}$ Crude odds ratio; ${ }^{2}$ Adjusted odds ratio

Reproductive health related factors: The majority of cases 51(98.3\%) and controls $110(91.7 \%)$ had experienced at least one full term pregnancies in lifetime. Fourteen $(23.3 \%)$ cases and $30(25.0 \%)$ controls had history of three to four live deliveries and 40(66.7\%) cases, and $34(28.3 \%)$ controls had more than four live deliveries (grand multiparous). More cases $2(28.3 \%)$ were above 25 years at the time of first full term delivery than the controls, 9(7.5\%)), $(\mathrm{OR}=3.7 ; 95 \%$ C.I. $=1.89-15.3)$. About a third of cases, 20(33.3\%), and controls, 41(34.2\%), reported history of nonviable pregnancy (abortion or stillbirth) during their reproductive lives. Postmenopausal status of participants, however, showed more than three times contribution to cervical cancer risk than nonmenopausal women $(\mathrm{OR}=3.3$, 95\% C.I.: 1.1-9.2). Only 7(11.7\%) of the study participants and $58(48.3 \%)$ of the controls never heard of cervical cancer. Similarly, two $(3.3 \%)$ cases and 7(5.8\%) controls had had history of PAP smear tests done in the studied population (Table 3). Those variables that were significant in the bivariate analyses were considered further in the multivariable analyses by use of logistic regression identified the number of full term pregnancies and age at full term pregnancies as independent predictor variables by performing [backward stepwise (likelihood ratio)] (Table 4). 
Table 2: Reproductive related variables and cervical cancer, JUSH

\begin{tabular}{|c|c|c|c|c|c|}
\hline Variables & Cases (\%) & Control (\%) & COR $(95 \% \mathrm{CI})$ & $\operatorname{AOR}(95 \% \mathrm{CI})$ & P-values \\
\hline \multicolumn{6}{|c|}{ No of term pregnancy } \\
\hline $1-2$ & $5(8.3)$ & $44(36.7)$ & 1.00 & 1.00 & \multirow[t]{3}{*}{0.000} \\
\hline $3-4$ & $14(23.3)$ & $30(25.0)$ & 4.1(1.3-12.6) & $4.7(0.8-27.2)$ & \\
\hline$>4$ & $40(66.7)$ & $34(28.3)$ & $10.3(3.6-29.0)$ & $12.4(2.4-64.2)$ & \\
\hline \multicolumn{6}{|l|}{ Nonviable delivery } \\
\hline No & $40(66.7)$ & $79(65.8)$ & 1.00 & 1.00 & \\
\hline Yes & $20(33.3)$ & $41(34.2)$ & $1.0(0.5-2.0)$ & $1.8(0.7-5.0)$ & \\
\hline \multicolumn{6}{|c|}{$\begin{array}{l}\text { Age in years at } 1^{\text {st }} \text { full } \\
\text { term delivery }\end{array}$} \\
\hline$<20$ & $21(35.0)$ & $73(60.9)$ & 1.00 & 1.00 & \multirow[t]{3}{*}{0.000} \\
\hline $20-25$ & $15(25.0)$ & $25(20.8)$ & $2.0(0.9-4.6)$ & $2.0(0.7-5.7)$ & \\
\hline$>25$ & $23(38.3)$ & $9(7.5)$ & $8.8(3.5-22.0)$ & $3.7(1.8-15.3)$ & \\
\hline \multicolumn{6}{|l|}{ History of STI } \\
\hline Yes & $5(8.3)$ & $22(15.0)$ & 1.00 & 1.00 & \\
\hline No & $55(91.7)$ & $98(81.7)$ & $2.4(0.8-6.8)$ & $6.4(1.2-33.6)$ & \\
\hline Unknown & $7(11.7)$ & $39(32.5)$ & $.32(.1-.81)$ & $0.3(0.09-1.0)$ & \\
\hline \multicolumn{6}{|c|}{$\begin{array}{l}\text { Age in years of } 1 \mathrm{st} \\
\text { sexual intercourse }\end{array}$} \\
\hline$<15$ & $06(10.0)$ & $28(23.3)$ & 1.00 & 1.00 & \\
\hline $15-20$ & $42(70.0)$ & $84(70.0)$ & $.48(.18-1.2)$ & $.21(.06-.75)$ & \\
\hline $21-30$ & $11(18.3)$ & $3(2.5)$ & $1.9(.49-7.9)$ & $.61(.09-3.9)$ & \\
\hline$>30$ & $1(1.7)$ & $5(4.2)$ & $.2(.02-2.2)$ & $.17(.01-3.5)$ & \\
\hline \multicolumn{6}{|l|}{ Age of menarche } \\
\hline$<15$ & $23(38.3)$ & $54(45.0)$ & 1.00 & 1.00 & \\
\hline$\geq 15$ & $21(35.0)$ & $50(41.7)$ & $.9(.4-1.9)$ & $1.0(0.4-2.5)$ & \\
\hline \multicolumn{6}{|l|}{ Menopausal status } \\
\hline Non menopausal & $23(38.3)$ & $96(80.0)$ & 1.00 & 1.00 & \\
\hline Menopausal & $37(61.7)$ & $24(20.0)$ & $6.4(3.2-12.7)$ & $3.3(1.1-9.2)$ & \\
\hline
\end{tabular}

Table 3: Knowlege about cervical cancer and history of PAP smear, JUSH

\begin{tabular}{lllll}
\hline Variables & Characteristics & Cases (\%) & Controls (\%) & P-value \\
\hline Heard of cancer? & Yes & $7(11.7)$ & $58(48.3)$ & \\
& No & $53(88.3)$ & $62(51.7)$ & \\
$\begin{array}{l}\text { Did you have history of } \\
\text { PAP smear done anywhere? }\end{array}$ & Yes & $2(3.3)$ & $7(5.8)$ & \\
& No & $58(96.7)$ & $113(94.2)$ & P-value = 0.000 \\
If not tested, & Not feeling & $17(28.3)$ & $21(17.5)$ & \\
why? & at risk & & \\
& No symptoms & $2(3.3)$ & $28(23.3)$ & \\
& Fear of vaginal & $1(1.7)$ & $7(5.8)$ & \\
& exam & & & \\
& Lack of interest & $6(10.0)$ & $10(8.3)$ & \\
& Unavailability & $13(21.7)$ & $7(5.8)$ & \\
& of test & & & \\
& Ignorance & $19(31.7)$ & $40(33.3)$ & \\
\hline
\end{tabular}


Table 4: Independent predictors of invasive cervical carcinoma, JUSH

\begin{tabular}{lclll}
\hline \multicolumn{1}{c}{ Variables } & Cases (\%) & Controls (\%) & p- values & AOR (95\% CI) \\
\hline $\begin{array}{l}\text { Numbers of full } \\
\text { pregnancies }\end{array}$ & & & & \\
$1-2$ & $5(8.3)$ & $44(36.7)$ & - & 1 \\
$3-4$ & $14(23.3)$ & $30(25.0)$ & 0.05 & $5.4(95 \%$ CI; $1.0-30.5)$ \\
$>4$ & $40(66.7)$ & $34(28.3)$ & 0.01 & $8.2(95 \%$ CI; $1.5-42.6)$ \\
Age at first full term pregnancy & & & & \\
$<20$ & $21(35.0)$ & $73(60.9)$ & - & 1 \\
$20-25$ & $15(25.0)$ & $25(20.8)$ & 0.16 & $3.1(95 \%$ CI; $0.74-5.9)$ \\
$>25$ & $23(38.3)$ & $9(7.5)$ & 0.02 & $4.4(95 \%$ CI; $1.2-16.4)$ \\
\hline
\end{tabular}

\section{DISCUSSION}

This study can hint cervical cancer as an emerging public health problem in Ethiopia. Most people remain unaware of how they can reduce their modifiable risk of developing cervical cancers and very little has been done to change this by public health agencies in the country. Public health action could help prevent invasive cervical cancers by health education, early detection and treatment and in due time by vaccination so that future generations could be salvaged from the growing threat of invasive cervical cancer. Overall, advancing age, husbands' life time sexual partners, lack of awareness to cervical cancer and PAP smear tests as well as grand multiparty ( $>4$ childbirth) and the age at first full term pregnancy (>25 years) were identified as significant risk factors that contributed to the advancement of cervical carcinoma in our patients. And, the latter two were the independent predictor risk factors for the development of invasive cervical cancers in this study where these two factors could be speculated to co-function to narrow the intervals among successive childbirths and risk our patients to help develop invasive cervical cancers in our communities. However, this hypothesis needs to be verified in prospective studies.

The major limitation of this study was failure to detect noninvasive cervical cancers (carcinoma in-situ) with the help of PAP smear tests. The test was not performed for lack of the necessary reagents and has never been practised in the southwestern part of the country. In one way or the other, performing the test per se is not very important for this study because it focused on invasive cervical carcinomas that present overwhelmingly with polypoid or ulcerative gross morphologic patterns.

This case control study depicted 47.7 years as a mean age for the cases where three quarters of them were older than 40 years of age. Similar studies described comparable mean ages, i.e. 46.7 years $(7,8)$. In most countries, cervical cancer risk maximizes at about 50 years of age; however, the age maximum incidence may be rather late in African populations to 50-65 years $(7,8)$. About two-third of our study participants came from relatively rural parts of the country, and similarly, $60 \%$ to $70 \%$ of women in sub-Saharan Africa who developed cervical cancer live in the rural areas $(2,9)$.

A husband with history of multiple wives was associated with cervical cancer in this study, and it was documented that sexual behavior of the male partners could be an important risk factor for cervical cancer with marginal significance (10). Moreover, women having three or four lifetime sexual partners were at a significant increased risk to cervical cancer compared to those reporting one or none partner (10).

The median incomes for the cases and controls were not found to be statistically significant in this study. Income level was not related to cervical cancer, and income level may be more likely to be misreported (11). Thus, the relation between the socioeconomic status and cervical cancer is still controversial. Some studies have shown that there is relationship between socioeconomic status and cervical cancer; nevertheless, others did not report any relationship between them (12). This study was in agreement with the latter findings. 
Cervical cancer awareness was very low in this study because an overwhelming majority of the study participants could not respond to questions to cervical cancer awareness. This finding hinted a very wide gap on cancer awareness in our set-up. In 2004 in Lagos, 81.7\% of 139 patients of advanced cervical cancer had never heard of cervical cancer before. Almost all (98.0\%) believed that their advanced disease was curable. Similar studies in Kenya and Tanzania as well as in USA also reported very poor knowledge of the disease in patients (13-16). In this study, two-third of cases and nearly one-third of controls had history of full term viable deliveries in excess of four children, and this was more than 8 times more risky to develop cervical cancer than those that have one or two children. Eight case control studies on invasive cervical cancers from four continents suggest that compared to women who had never given birth, those with three or more full term pregnancies had 2.6 times the risk of developing cervical cancer while women with seven or more births had 3.8 times the risk (17). Different studies depicted that multiparty is strongly related to the development of cervical cancer. The effects of parity in cervical cancer development seem to be mainly detectable in countries with high fertility rates and are rarely found in low parity countries. Parity is probably a good marker of normal estrogen environment throughout the fertile years of women as well as a marker of repeated cervical trauma among women with multiparity $(12,18,19)$.

Age of first full term delivery in women older than 25 years of age was related to the development of cervical cancer and this may suggest that it is not only the age of first full term delivery that may matter most but the intervals among deliveries might be important for the disposition of cervical cancer. Related research finding uncovered that women who married late but gave birth to a large number of children were generally found to be suffering from cervical cancer. These results support the hypothesis that it is not so much the number of parity per se that enhances the risk, but the rapidity of multiple pregnancies that matters. These findings warrant serious consideration in the future studies given the obvious implications for prevention (13).

In conclusion, poor knowledge of cervical cancer was observed that required more work to be done to increase knowledge of mothers on cervical cancer and associated risk factors. Behavioral communication activities and establishment of cervical cancer screening programs for the young could help reduce the advancement of cervical cancer particularly among the less knowledgeable, older and grand multiparous women in our part of the world.

\section{ACKNOWLEDGEMENT}

We extended our most sincere gratitude to Jimma University for the financial support and Jimma University Specialized Hospital, the data collectors, the supervisor and the study participants for their time, support and courtesy. Our special thanks also go to Ato Tariku Dejene and Dr Wondwossen Kassahun for their extraordinary remarks during data entry and analyses.

\section{REFERENCES}

1. Lingwood RJ, Boyle P, Milburn A et al.The challenge of cancer control in Africa. Nature reviews cancer. 2008; 8: 389-403.

2. Anorlu RI. Cervical cancer: the sub-Saharan African perspective. Reproductive Health Matters 2008;16(32):41-49).

3. Loutfi, A. \& Pickering, J.L. The distribution of cancer specimens from two pathology centers in Ethiopia.Ethiop.Med.J.1992; 30:1317.

4. WHO, Report on cancer, Geneva, 2009.

5. Central Statistics Autority; Census: Oromia region, Ethiopia, 2007.

6. Rubin R, Strayer SD- Neoplasia In: Giordano A, De Falco G, Rubin E, Rubin R eds. Rubins Pathology: Clinocopathologic basis of medicine 5th ed. Philadelphia, lippincott williams. Page 143-148.

7. Brinton LA, Reeves WC, Brenes MM, et al. Parity as a risk factor for cervical cancer. American Journal of Epidemiology. 1989; 130 (3): 486-496.

8. Biswas LN, Manna B, Miati PK, Sengupta S. Sexual risk factors for cervical cancer among rural Indian women: A case control study. International Journal of Epidemiology. 1997; 26(3): 491-495.

9. Walraven G, Scherf C, West B et al. The burden of reproductive organ diseases in rural 
women in Gambia, West Africa. The LANCET. 2001; 357 (9263): 1161-1167.

10. Robert N, John Z, Delphine C; et al. A case control study of cancer of the uterine cervix in Uganda. Europian Journal of cancer prevention. 2007;16(6): 13-19.

11. San Jose SD, Bosch FX, Munoz N, et al. Socioeconomic differences in cervical cancer: Two case control studies in Colombia and Spain. Am, J. Public Health. 1997; 86: 15321538.

12. Taherian AA, Fatahi E, Soleimani B. Study of risk factors for cervical cancer. A case control study in Isfahan-Iran. Kuwait medical Journal. 2002; 34 (2): 128-132.

13. Green J, De Gonzales AB, Sweetland S, Beral $\mathrm{V}$, Chilvers C, chrossley B, deacon J, hermon $\mathrm{C}$, Jha $\mathrm{P}$, mant $\mathrm{D}$, pito j, pike M, vessey MP. Risk factors for carcinomas of cervix in women aged 20-44years. British Journal of cancer 2003; 89: 2078-2086.

14. Hasenyager C. Knowledge of cervical cancer attending a University health centre. Journal of American college Health. 1999; 47 (5): 221-224.

15. Tebeu P-M, Major AL, Rapiti E, et al.The attitude and knowledge of cervical cancer by
Cameronian women; a clinical survey conducted in Maroua, the capital of Far North Province of Cameroon. International Journal of gynecologic cancer. 2008; 18 (4): 761-765.

16. Nwankwo KC., Aniebue UU., Aguwa EN, et al. Knowledge attitudes and practices of cervical cancer screening among urban and rural Nigerian women: a call for education and mass screening. European Journal of Cancer Care. 2010; 20: 362-367.

17. Plummer M, Appley $\mathrm{P}$, Beral V, et al. Cervical Carcinoma and Sexual Behavior: Collaborative Reanalysis of Individual Data on 15,461 Women with Cervical Carcinoma and 29,164 Women without Cervical Carcinoma from 21 Epidemiological Studies.Cancer Epidemiol Biomarkers Prev 2009;18(4):1060-9).

18. Brinton LA, Reeves WC, Brenes MM, et al. Parity as a risk factor for cervical cancer. American Journal of Epidemiology. 1989; 130 (3): 486-496.

19. Bayo S, Bosch FX, de Sanjose S, et al. Risk factors of invasive cervical cancer in Mali. International Journal of Epidemiology. 2002 ; $31: 202-209$. 